\title{
Goldman's Type Theorem for Index 3
}

By

\author{
Masaki IZUMI*
}

\begin{abstract}
We show a Goldman's type theorem for any inclusions of (not necessarily AFD) factors with the principal graph $A_{5}$. Our main tools are correspondences and sectors.
\end{abstract}

\section{§1. Introduction and Main Results}

In our previous paper [I, Theorem 5.1], we showed that any pair of $\mathrm{III}_{1}$ AFD factors whose principal graph is the Coxeter graph $A_{5}$ is as follows.

$$
R_{\infty} \rtimes S_{3} \supset R_{\infty} \rtimes S_{2}
$$

where $R_{\infty}$ is the type $\mathrm{III}_{1}$ AFD factor. This theorem has been known in the case of the type $\mathrm{II}_{1}$ and $\mathrm{III}_{\lambda}(0<\lambda<1)$ AFD factors using classifications of subfactors $[\mathrm{Li}, \mathrm{O}, \mathrm{P}]$. The aim of the present work is to generalize this theorem for arbitrary factors. Since we will not use classifications of subfactors and group actions, we need no assumption of AFD.

V. Jones' index theory is one of the most important and interesting topics in recent operator algebras, and many connections with other areas of mathematics and mathematical physics are pointed out. As one of his motivations of his definition of index [J], there is Goldman's theorem [G], which says that any inclusion of factors with index 2 is written by the crossed product of a $\mathbb{Z}_{2}$ action. One direction of generalizations of this theorem is a characterization of crossed products in terms of indices and higher relative commutants (principal graphs). This was done by M. Pimsner and S. Popa [PP1], A. Ocneanu in the case of $\mathrm{II}_{1}$ factors, and $\mathrm{H}$. Kosaki [K2] in the case of properly infinite factors. Our approach is considered to be another direction of the generalizations.

Communicated by H. Araki, December 11, 1991.

1991 Mathematics Subject Classification: 46L37

* Research Institute for Mathematical Sciences, Kyoto University, 606, Japan. 
In Section 3, we first prove the main theorem in the case of properly infinite factors using sectors, and next in the case of finite factors using correspondences. Although the latter argument may be valid for general cases, we leave the former because of perspective.

Our basic references are [G.H.J, K1] for the index theory and [I, L2] for the sector theory.

The author would like to thank $\mathbb{H}$. Araki for his constant encouragement and $\mathbb{H}$. Kosaki for discussions.

\section{§ 2. Preliminaries}

In this section we extend the notion of sectors defined by $\mathbb{R}$. Longo in [L2], and show basic facts.

Throughout this paper we assume that von Neumann algebras have separable preduals and Hilbert spaces are separable.

Let $M$ and $N$ be factors. We use the following notations.

$\operatorname{Mor}(N, M)$ : the set of unital normal isomorphisms from $N$ into $M$.

In the case of $N \subset M$,

$E(M, N)$ : the set of faithful normal conditional expectations from $M$ to $N$.

$\rho_{1}, \rho_{2} \in \operatorname{Mor}(N, M)$ are unitary equivalent if and only if there exists a unitary $u \in M$ such that

$$
\rho_{1}=\operatorname{Ad}(u) \cdot \rho_{2} .
$$

For properly infinite factors $M, N$, we denote by $\operatorname{Sect}(M, N)$ the quotient of $\operatorname{Mor}(N, M)$ by the above equivalence, and call elements in $\operatorname{Sect}(M, N) M-N$ sectors. If $\rho \in \operatorname{Mor}(N, M),{ }_{M}[\rho]_{N}$ denotes its class in $\operatorname{Sect}(M, N)$. If $M=N$ $\operatorname{Sect}(M, N)$ coincides with $\operatorname{Sect}(M)$ in $[\mathrm{L} 2, \mathbb{I}]$. We sometimes associate different sectors with one morphism. For example, let $N$ be a subfactor of $M$ and $\gamma: M \rightarrow N$ the canonical endomorphism [L3]. If we consider $\gamma$ a map from $M$ to $N$ (resp. from $M$ to $M$, from $N$ to $M$, from $N$ to $N$ ), we write ${ }_{N}[\gamma]_{M}$ (resp. ${ }_{M}[\gamma]_{M},{ }_{M}[\gamma]_{N},{ }_{N}[\gamma]_{N}$ ). Sum, product and irreducible decomposition are defined on the sectors in the same way as in $[\mathbb{I}, \S 2]$, except that the product is a map $\operatorname{Sect}(M, N) \times \operatorname{Sect}(N, \mathbb{L}) \rightarrow \operatorname{Sect}(M, L)$.

Let $L, M, N$ be properly infinite factors and $H$ be $L-M$ correspondence (For the definition of correspondence see $[\mathrm{P} 2, \mathrm{~L} 2, \mathbb{1}]$.). If $\rho \in \mathbb{M} \operatorname{or}(N, M)$ (resp. $\rho \in \operatorname{Mor}(N, L))$, we define a $L-N$ correspondence $H_{\rho}$ (resp. $N-M$ correspondence ${ }_{\rho} H$ ) by

$$
\begin{gathered}
x \cdot \xi^{\prime} \cdot y \equiv x \cdot \xi \cdot \rho(y), \quad x \in L, \quad y \in N, \quad \xi \in \mathbb{H}, \quad \xi^{\prime} \in H_{\rho} \\
\text { (resp. } \left.x \cdot \xi^{\prime} \cdot y \equiv \rho(x) \cdot \xi \cdot y, \quad x \in N, \quad y \in M, \quad \xi \in \mathbb{H}, \quad \xi^{\prime} \in{ }_{\rho} H\right)
\end{gathered}
$$


where $\xi=\xi^{\prime}$ as an element of the Hilbert space. If we consider $\mathrm{L}^{2}(M)$ as $M-M$ correspondence by the natural actions, then the map $\rho \mapsto \mathrm{L}^{2}(M)_{\rho}$ induces bijection between $\operatorname{Sect}(M, N)$ and the set of equivalence classes of $M-N$ correspondence [L2, Corollary 2.2]. (Note that our convention is different from that in $[\mathrm{L} 2, \mathrm{I}]$, because it is convenient to consider that the left action is standard.) We define a map called conjugation ${ }_{M}[\rho]_{N} \mapsto_{N} \overline{[\rho]}_{M}$ using that of the correspondences and the above bijection. For simplicity $\bar{\rho}$ denotes one of representatives of ${ }_{N} \overline{[\rho]}_{M}$. Let $\gamma: M \rightarrow \rho(N)$ be the canonical endomorphism. Then $\bar{\rho}$ is given as follows [L2, Theorem 3.1].

$$
\bar{\rho}=\rho^{-1} \cdot \gamma \text {. }
$$

Using similar calculation in the proof of [L2, Theorem 3.1], we can show the following relation.

$$
{ }_{M}\left(\mathrm{~L}^{2}(M)_{\rho}\right)_{N} \cong{ }_{M}\left({ }_{\bar{\rho}} \mathrm{L}^{2}(N)\right)_{N} .
$$

The following proposition shows that our convention is natural.

Proposition 2.1. Let $M, N, L$ be properly infinite factors and ${ }_{M}\left[\rho_{1}\right]_{N} \in$ $\operatorname{Sect}(M, N),{ }_{N}\left[\rho_{2}\right]_{L} \in \operatorname{Sect}(N, L)$. Then,

$$
{ }_{M}\left(\mathrm{~L}^{2}(M)_{\rho_{1}}\right)_{N} \otimes_{N}{ }_{N}\left(\mathrm{~L}^{2}(N)_{\rho_{2}}\right)_{L} \cong{ }_{M}\left(\mathrm{~L}^{2}(M)_{\rho_{1} \rho_{2}}\right)_{L}
$$

where $\underset{N}{\otimes}$ means Sauvageot's relative tensor product $[\mathrm{S}]$.

Proof. Due to (2.1) and

we have

$$
\left.{ }_{N} \mathrm{~L}^{2}(N)\right)_{N} \otimes_{N}{ }_{N} \mathrm{~L}^{2}(N)_{N} \cong{ }_{N} \mathrm{~L}^{2}(N)_{N},
$$

$$
\begin{aligned}
{ }_{M}\left(\mathrm{~L}^{2}(M)_{\rho_{1}}\right)_{N} \otimes_{N}{ }_{N}\left(\mathrm{~L}^{2}(N)_{\rho_{2}}\right)_{L} & \cong{ }_{M}\left(\bar{\rho}_{1} \mathrm{~L}^{2}(N)\right)_{N} \bigotimes_{N}{ }_{N}\left(\mathrm{~L}^{2}(N)_{\rho_{1}}\right)_{L} \\
& \cong{ }_{M}\left(\bar{\rho}_{1} \mathrm{~L}^{2}(N)_{\rho_{2}}\right)_{L} \cong{ }_{M}\left(\mathrm{~L}^{2}(M)_{\rho_{1} \rho_{2}}\right)_{L} \text {. Q.E.D. }
\end{aligned}
$$

Remark 2.2. If $M$ is a $\mathbb{I I}_{1}$ factor we cannot use sectors. But (2.1) and Proposition 2.1 make sense if we consider only automorphisms. Namely, the following hold.

$$
\begin{gathered}
{ }_{M}\left(\mathrm{~L}^{2}(M)_{\rho}\right)_{M} \cong{ }_{M}\left({ }_{\rho^{-1}} \mathrm{~L}^{2}(M)\right)_{M} \\
{ }_{M}\left(\mathrm{~L}^{2}(M)_{\rho_{1}}\right)_{M}{ }_{M}^{\otimes}{ }_{M}\left(\mathrm{~L}^{2}(M)_{\rho_{2}}\right)_{M} \cong{ }_{M}\left(\mathrm{~L}^{2}(M)_{\rho_{1} \rho_{2}}\right)_{M}
\end{gathered}
$$

where $\rho, \rho_{1}, \rho_{2} \in \operatorname{Aut}(M)$ and we use $\bar{\rho}=\rho^{-1}$.

We assume again that $M$ and $N$ are properly infinite factors. Let $\phi: M \rightarrow N$ be a unital normal completely positive map and $\Omega$ be a separating and cyclic vector for $N$. Then there exists a unique sector ${ }_{N}[\rho]_{M} \in \operatorname{Sect}(N, M)$ and $\xi \in$ $\mathrm{L}^{2}(N)$ satisfying the following [I, subsection 2.2].

$$
\begin{gathered}
\left\langle x J_{N} \phi\left(y^{*}\right) J_{N} \Omega, \Omega\right)=\left\langle x J_{N} \rho\left(y^{*}\right) J_{N} \xi, \xi\right\rangle \quad \text { for } x \in N, y \in M \\
\overline{N J_{N} \rho(M) J_{N} \xi}=\mathrm{L}^{2}(N)
\end{gathered}
$$


where $J_{N}$ is the modular conjugation of $N$. We denote ${ }_{N}[\rho]_{M}$ by ${ }_{N}[\phi]_{M}$. If $M \supset N$ and $E \in \mathrm{E}(M, N)$, we can compute ${ }_{N}[E]_{M}$ explicitly as in [L2, Proposition 3.3], [I, subsection 2.2]. Let $\gamma: M \rightarrow N$ be the canonical endomorphism. Then,

$$
{ }_{N}[E]_{M}={ }_{N}[\gamma]_{M} .
$$

If we consider $E$ to be a map from $M$ to $M$, we obtain

$$
{ }_{M}[E]_{M}={ }_{M}[\gamma]_{M} .
$$

Now we will move to the index theory. Let ${ }_{M}[\rho]_{N} \in \operatorname{Sect}(M, N)$. We define the (statistical) dimension $d(\rho)$ by

$$
d(\rho)=[M: \rho(N)]_{0}^{1 / 2} .
$$

In the above equation $[M: \rho(N)]_{0}$ means Hiai's minimum index $[\mathrm{H}]$. If there is possibility of confusion we will write $d\left({ }_{M} \rho_{N}\right)$. As in [I, subsection 2.3] $d(\rho)$ satisfies the additivity and the multiplicativity [KL], [L4].

In the same way as in [L2, Theorem 4.1], [I, Theorem 2.1], we can show the following proposition.

Proposition 2.3. Let $M, N$ be a pair of properly infinite factors and ${ }_{N}\left[\rho_{1}\right]_{M} \in \operatorname{Sect}(N, M){ }_{M}\left[\rho_{2}\right]_{N} \in \operatorname{Sect}(M, N)$ with $d\left(\rho_{1}\right), d\left(\rho_{2}\right)<\infty$. If $\rho_{1}$ and $\rho_{2}$ are irreducible then the following conditions are equivalent:

(1) ${ }_{N}\left[\rho_{1} \rho_{2}\right]_{N}$ or ${ }_{M}\left[\rho_{2} \rho_{1}\right]_{M}$ contains the identity sector.

(2) ${ }_{N}\left[\rho_{2}\right]_{M}={ }_{N}\left[\rho_{1}\right]_{M}$.

If $\rho_{1}$ and $\rho_{2}$ satisfy the above condition, ${ }_{N}\left[\rho_{1} \rho_{2}\right]_{N}$ and ${ }_{M}\left[\rho_{2} \rho_{1}\right]_{M}$ contain the identity sector with multiplicity one.

Let $M \supset N$ be a pair of properly infinite factors and $\gamma: M \rightarrow N$ be the canonical endomorphism. Then,

$$
M \supset N \supset \gamma(M) \supset \gamma(N) \supset \gamma^{2}(M) \supset \cdots
$$

is the tower associated with $M \supset N$. Let $\imath: N \rightarrow M$ be the embedding map. By easy computation we obtain

$$
{ }_{N} \overline{[l]}_{M}={ }_{N}[\gamma]_{M} .
$$

Then the principal graphs of $M \supset N$ and $N \supset \gamma(M)$ show the fusion rules of the following sequences of sectors $[I, \S 3]$.

$$
\begin{aligned}
{ }_{M}[l]_{N}, & { }_{M}[l]_{N} \cdot{ }_{N}[\gamma]_{M}={ }_{M}[\gamma]_{M}, & \left.\left.{ }_{M}[l]_{N} \cdot{ }_{N}[\gamma]\right]_{M}{ }^{\cdot}[l]\right]_{N}={ }_{M}[\gamma]_{N} \cdots \\
{ }_{N}[\gamma]_{M}, & \left.{ }_{N}[\gamma]_{M} \cdot{ }_{M}[l]\right]_{N}={ }_{N}[\gamma]_{N}, & { }_{N}[\gamma]_{M} \cdot{ }_{M}[l]_{N} \cdot{ }_{N}[\gamma]_{M}={ }_{N}\left[\gamma{ }^{2}\right]_{M} \cdots .
\end{aligned}
$$

We call the sectors appearing in the decomposition of the above sectors as descendant sectors. 
Before closing this section, we show the following proposition as an application of sectors.

Proposition 2.4. Let $M \supset N$ be a pair of properly infinite factors with finite depth. If the principal graph of $M \supset N$ has two points whose entries of the Perron-Frobenius eigenvector are minimal and they have different colors, (note that principal graphs are always bipartite), then $M$ is isomorphic to $N$.

Proof. Let ${ }_{M}[\rho]_{N}$ be the descendant sector corresponding to one of the above points which is not the distinguished point. Then the above condition implies $d(\rho)=1$ as in [I, subsection 2.3]. This means $[M: \rho(N)]=1$ and so we have the result.

Remark 2.5. If the principal graph has a graph automorphism which does not preserve the colors of vertices, then the condition of Proposition 2.4 is satisfied. For example, Coxeter graph $A_{\text {even }}$ and $D_{\text {odd }}^{(1)}$ satisfy the condition.

\section{§3. The Main Theorem}

The main theorem of the present work is as follows.

Theorem 3.1. Let $M \supset N$ be a pair of factors with the principal graph $A_{5}$. Then there exists $L$ a subfactor of $N$ and an outer action of $S_{3}$ on $L$ such that

$$
(M \supset N) \cong\left(L \rtimes S_{3} \supset L \rtimes S_{2}\right) .
$$

Proof. First, we show the theorem in the case of properly infinite factors using sectors. Let $\gamma: M \rightarrow N$ be the canonical endomorphism and $\imath: N \rightarrow M$ the embedding map. The descendant sectors and fusion rules are as follows (see Fig. 1) [I, §5]:

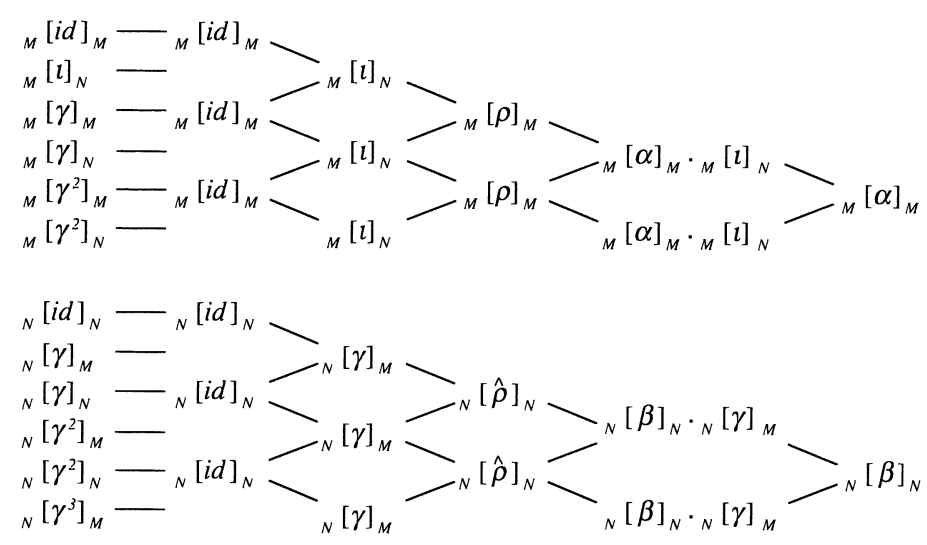

Fig. 1. The diagram of fusion rules of $A_{5}$. 


$$
\begin{aligned}
{ }_{M}[\gamma]_{M} & ={ }_{M}[i d]_{M} \oplus{ }_{M}[\rho]_{M}, \\
{ }_{M}[\rho]_{M}{ }_{M}[l]_{N} & ={ }_{M}[l]_{N} \oplus{ }_{M}[\alpha]_{M}{ }^{\cdot{ }_{M}}[l]_{N}, \\
{ }_{M}[\rho]_{M}{ }^{2} & ={ }_{M}[i d]_{M} \oplus{ }_{M}[\alpha]_{M} \oplus{ }_{M}[\rho]_{M}, \\
\left.{ }_{M}[\alpha]_{M}{ }^{\cdot}[\rho]\right]_{M} & ={ }_{M}[\rho]_{M} \cdot{ }_{M}[\alpha]_{M}={ }_{M}[\rho]_{M}, \\
d(\rho) & =2, \quad d(\alpha)=1 . \\
{ }_{N}[\hat{\rho}]_{N}{ }^{\cdot}{ }_{N}[\gamma]_{M} & ={ }_{N}[\gamma]_{M} \oplus{ }_{N}[\beta]_{N} \cdot{ }_{N}[\gamma]_{M}, \\
{ }_{N}[\hat{\rho}]_{N}{ }^{2} & ={ }_{N}[i d]_{N} \oplus{ }_{N}[\beta]_{N} \oplus{ }_{N}[\hat{\rho}]_{N}, \\
{ }_{N}[\beta]_{N} \cdot{ }_{N}[\hat{\rho}]_{N} & ={ }_{N}[\hat{\rho}]_{N} \cdot{ }_{N}[\beta]_{N}={ }_{N}[\hat{\rho}]_{N}, \\
d(\hat{\rho}) & =2, \quad d(\beta)=1 .
\end{aligned}
$$

${ }_{M}[\rho]_{M},{ }_{N}[\hat{\rho}]_{N},{ }_{M}[\alpha]_{M},{ }_{N}[\beta]_{N}$ are self-conjugate and $d(\alpha)=d(\beta)=1$ implies $\alpha \in \operatorname{Aut}(M), \beta \in \operatorname{Aut}(N)$. We can choose $\alpha$ and $\beta$ such that $\alpha^{2}=i d_{M}, \beta^{2}=i d_{N}$ as in [I, Proposition 3.3]. Now we will consider the following inclusions of factors.

$$
M \supset N \supset L \equiv N^{\beta}
$$

where $N^{\beta}$ is the fixed point algebra of $N$ under $\beta$. Let $E_{1}, E_{2}$ be the unique elements in $\mathrm{E}(M, N)$ and $\mathrm{E}(N, L)$. Due to [L1, Proposition 5.1] there exists an isometry $v \in N$ satisfying the following.

$$
\begin{array}{cc}
v y=\gamma(y) v \quad \text { for } \quad y \in N \\
E_{1}(x)=v^{*} \gamma(x) v \quad \text { for } \quad x \in M .
\end{array}
$$

If we define $E \in \mathbb{E}(M, N)$ by $E=E_{2} \cdot E_{1}$, we can show the following as in [I, $\S 5]$.

$$
{ }_{M}[E]_{M}={ }_{M}[\gamma]_{M} \oplus_{M}[\beta \cdot \gamma]_{M}
$$

From Fig. 1 and

$$
\left.{ }_{N}\left[\gamma^{2}\right]_{M}={ }_{N}[\gamma]_{M} \cdot{ }_{M}[l]\right]_{N} \cdot{ }_{N}[\gamma]_{M}=\overline{\left.\left({ }_{M}[l]_{N} \cdot{ }_{N}[\gamma]_{M} \cdot{ }_{M}[l]\right]_{N}\right)},
$$

we can see

$$
{ }_{N}[\beta \cdot \gamma]_{M}=\overline{\left({ }_{M}[\alpha]_{M} \cdot{ }_{M}[l]_{N}\right)}={ }_{N}[\gamma]_{M} \cdot{ }_{M}[\alpha]_{M}
$$

where we use (2.6) and self-conjugacy of $\alpha$. So using (3.1), (3.4) we have

$$
\begin{aligned}
{ }_{M}[E]_{M} & ={ }_{M}[\gamma]_{M} \oplus_{{ }_{M}}[\gamma]_{M} \cdot{ }_{M}[\alpha]_{M} \\
& ={ }_{M}[i d]_{M} \oplus_{M}[\alpha]_{M} \oplus 2{ }_{M}[\rho]_{M} .
\end{aligned}
$$




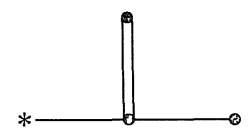

Fig. 2. A part of the principal graph of $M \supset L$

If the depth of $M \supset L$ is 2, we obtain the result due to [I, Corollary 5.4, Proposition 4.1]. Although [I, Proposition 4.2] is also valid for our case, we apply another proof for latter use. Due to (2.5), (3.11) means the principal graph of $M \supset L$ contains the graph in Fig. 2 as a part, and it is easy to see that the Perron-Frobenius eigenvalue of this graph is $\sqrt{6}$. On the other hand, thanks to $[\mathrm{KL}, \mathrm{L} 4]$ we have $[M: L]_{0}=[M: N]_{0}[N: L]_{0}=6$. So this graph must coincide with the principal graph of $M \supset L$ because of [GHJ, Lemma 1.4.2].

In the case of finite factors, we have to repeat the essentially same argument treating correspondences (bimodules) directly. Before completing the proof, we will prepare a few facts on correspondences. Let

$$
N \subset M \subset M_{1} \subset M_{2} \subset \cdots,
$$

be the tower associated with $N \subset M$. (For simplicity, we will write ${ }_{M}\left(M_{n}\right)_{M}$ instead of ${ }_{M} \mathrm{~L}^{2}\left(M_{n}\right)_{M}$.) Then it is well known that $M_{n}$ is equivalent to

$$
\overbrace{M \otimes \underset{N}{M} \underset{N}{\otimes} \cdots \underset{N}{\otimes} M}^{n+1},
$$

as an $M-M(N-M, M-N, N-N)$ correspondence [GHJ, Corollary 3.6.5] [J2]. The correspondences appearing in the irreducible decomposition of ${ }_{M}\left(M_{n}\right)_{M},{ }_{N}\left(M_{n}\right)_{M},{ }_{M}\left(M_{n}\right)_{N},{ }_{N}\left(M_{n}\right)_{N}$, and their restriction-induction graph are as in Fig. 3 (see [O2]). Using the formula in [J1, page 4] and the local index formula in [PP2, Corollary 3.2], we can show the following.

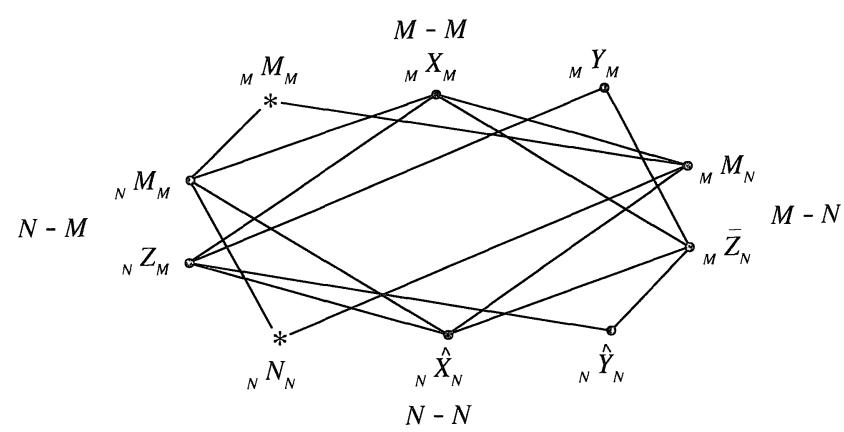

Fig. 3. The restriction-induction graph of $A_{5}$ 


$$
\begin{aligned}
& \operatorname{dim}_{M}(X)=\operatorname{dim}(X)_{M}=2, \\
& \operatorname{dim}_{M}(Y)=\operatorname{dim}(Y)_{M}=1, \\
& \operatorname{dim}_{N}(\hat{X})=\operatorname{dim}(\hat{X})_{N}=2, \\
& \operatorname{dim}_{N}(\hat{Y})=\operatorname{dim}(\hat{Y})_{N}=1 .
\end{aligned}
$$

Since $\left\{{ }_{M} M_{M},{ }_{M} X_{M},{ }_{M} Y_{M}\right\}$ and $\left\{{ }_{N} N_{N},{ }_{N} \hat{X}_{N},{ }_{N} \hat{Y}_{N}\right\}$ are closed under conjugation and ${ }_{M} M_{M},{ }_{N} N_{N}$ are self-conjugate, they are all self-conjugate. Due to (3.13), (3.15) and self-conjugacy of ${ }_{M} Y_{M},{ }_{N} \hat{Y}_{N}$, there exist outer automorphisms $\alpha \in$ $\operatorname{Aut}(M), \beta \in \operatorname{Aut}(N)$ satisfying

$$
\begin{gathered}
\alpha^{2} \in \operatorname{Int}(M) \quad \beta^{2} \in \operatorname{Int}(N), \\
{ }_{M} Y_{M} \cong{ }_{M}\left({ }_{\alpha} M\right)_{M} \cong{ }_{M}\left(M_{\alpha}\right)_{M}, \\
{ }_{N} \widehat{Y}_{N} \cong{ }_{N}\left({ }_{\beta} N\right)_{N} \cong{ }_{N}\left(N_{\beta}\right)_{N},
\end{gathered}
$$

where we use (2.2), (2.3). So using the restriction-induction graph, we have

$$
{ }_{N} Z_{M} \cong{ }_{N}\left(M_{\alpha}\right)_{M} \cong{ }_{N}\left({ }_{\beta} M\right)_{M} \cong{ }_{N}\left({ }_{\alpha} M\right)_{M} .
$$

Lemma 3.2. For each fixed $\beta, \alpha$ can be chosen such that

$$
\left.\alpha\right|_{N}=\beta \text {. }
$$

Proof. First we fix $\alpha$ and $\beta$. Due to (3.19) the following two $N-M$ actions on $\mathbb{L}^{2}(M)$ are equivalent.

$$
\begin{aligned}
& x \cdot \xi \cdot y \equiv \beta(x) J y^{*} J \xi \quad \text { for } \quad x \in N, \quad y \in M, \quad \xi \in \mathbb{L}^{2}(M) \\
& x \cdot \xi \cdot y \equiv \alpha(x) J y^{*} J \xi \quad \text { for } \quad x \in N, \quad y \in M, \quad \xi \in \mathbb{L}^{2}(M) \text {. }
\end{aligned}
$$

where $J$ is the canonical conjugation of $M$. Then there exists a unitary $w \in \mathbb{B}\left(\mathbb{L}^{2}(M)\right)$ satisfying

$$
\begin{array}{cc}
w J y^{*} J=J y^{*} J w & \text { for } \quad y \in M, \\
w \beta(x)=\alpha(x) w \quad \text { for } \quad & x \in N .
\end{array}
$$

From the first equation we obtain $w \in M$. So the second equation shows that $\operatorname{Ad}\left(w^{*}\right) \cdot \alpha$ is the desired automorphism.

Q.E.D.

As we computed the fusion rules of sectors, we can show the following.

$$
\begin{gathered}
{ }_{M} X_{M} \otimes_{M}^{\otimes}{ }_{M} X_{M} \cong{ }_{M} M_{M} \oplus_{M} X_{M} \oplus_{M}\left(M_{\alpha}\right)_{M}, \\
{ }_{M}\left({ }_{\alpha} X\right)_{M} \cong{ }_{M}\left(X_{\alpha}\right)_{M} \cong{ }_{M} X_{M}, \\
{ }_{N} \hat{X}_{N}{ }_{N}{ }_{N} \hat{X}_{N} \cong{ }_{N} N_{N} \oplus_{N} \hat{X}_{N} \oplus_{N}\left(N_{\beta}\right)_{N}, \\
{ }_{N}\left({ }_{\beta} \hat{X}\right)_{N} \cong{ }_{N}\left(\hat{X}_{\beta}\right)_{N} \cong{ }_{N} \hat{X}_{N} .
\end{gathered}
$$

As in the properly infinite case, the following holds. 
Lemma 3.3. We can take $\beta$ such that $\beta^{2}=i d_{N}$.

Proof. Let $P \equiv \operatorname{Hom}\left(\hat{X}_{N}\right)$. Then $N$ can be considered a subfactor of $P$. Due to (3.23), $\beta$ is implemented by a unitary in $P$. Thanks to $P \cap N^{\prime}=\mathrm{C}$ the obstruction of $\beta$ is trivial [C], and we obtain the result. Q.E.D.

We assume $\beta^{2}=i d_{N}$ and $\alpha \mid N=\beta$. Note that $\alpha^{2}=i d_{M}$ also holds due to (3.16) and $M \cap N^{\prime}=$ C. Let $\theta$ be the unique extension of $\alpha$ to $M_{1}$ such that $\theta\left(e_{N}\right)=e_{N}[\mathrm{Kw}][\mathrm{Li}]$. Then, since the principal graph of $M \subset M_{1}$ is $A_{5}$, [PP3, Proposition 1.7 (iii)] shows that $\theta$ is outer. As in the properly infinite case, we will consider the following inclusions of factors.

Let

$$
L \equiv N^{\beta} \subset N \subset M \subset M_{1} \subset M_{1} \underset{\theta}{\rtimes \mathbb{Z}_{2}} .
$$

$$
L \subset M \subset \tilde{M}_{1} \subset \tilde{M}_{2} \subset \cdots
$$

be the tower associated with $L \subset M$.

\section{Lemma 3.4.}

(i) $M \subset M_{1} \rtimes_{\theta} \mathbb{Z}_{2}$ is isomorphic to $M \subset \tilde{M}_{1}$

(ii) $M \subset \tilde{M}_{1}$ is an irreducible pair.

Proof. (i): Let $J$ be the canonical conjugation of $M$ and $u$ the canonical implementation of $\alpha$. By definition, we can see

$$
\begin{aligned}
\tilde{M}_{1} & =J L^{\prime} J=J\left(N^{\prime} \vee\{u\}\right) J \\
& =J N^{\prime} J \vee\{u\}=M_{1} \vee\{u\} .
\end{aligned}
$$

Since $\alpha$ preserves $N, \alpha$ commutes with the unique element in $\mathrm{E}(M, N)$. Thus $u$ commutes with $e_{N}$ and so $\left.\operatorname{Ad}(u)\right|_{M_{1}}=\theta$ holds. Thanks to [A, II.3] we obtain the result. (ii): If $x+y u \in \tilde{M}_{1} \cap M^{\prime}, x, y \in M_{1}$. Then $x \in \mathbf{C}$, and $y$ satisfies the following.

$$
y a=\alpha(a) y \quad \text { for } \quad a \in M .
$$

From $M_{1} \cap M^{\prime}=\mathbf{C}, y$ is a multiple of a unitary in $M_{1}$. But this implies $y=0$ because of [PP3, Proposition 1.7. (ii)].

Q.E.D.

Completion of the proof of Theorem 3.1. Due to Lemma 3.4 the irreducible decomposition of ${ }_{M}\left(\tilde{M}_{1}\right)_{M}$ is as follows.

$$
\begin{aligned}
&{ }_{M}\left(\tilde{M}_{1}\right)_{M} \cong{ }_{M}\left(M_{1}\right)_{M} \oplus_{M}\left(M_{1} u\right)_{M} \\
& \cong{ }_{M}\left(M_{1}\right)_{M} \oplus_{M}\left(\left(M_{1}\right)_{\alpha}\right)_{M} \\
& \cong{ }_{M} M_{M} \oplus_{M} X_{M} \oplus_{M}\left(M_{\alpha}\right)_{M} \oplus_{M}\left(X_{\alpha}\right)_{M} \\
& \cong{ }_{M} M_{M} \oplus_{M}\left(M_{\alpha}\right)_{M} \oplus 2_{M} X_{M} .
\end{aligned}
$$


In the above equations we used (3.21). Then as in the properly infinite case, the depth of $L \subset M$ is two. Thanks to [I, Corollary 5.4], the relation between $L$ and $M$ are one of the following. (i) $L \subset M \cong L \subset L \rtimes G$, (ii) $L \subset M \cong M^{G} \subset$ $M$, where $G$ is a group of order 6 . Suppose (ii). Due to the characterization of crossed product $\left[\mathrm{PP} 1\right.$, Corollary 1.1.6], $\operatorname{dim}\left(\tilde{M}_{2} \cap M^{\prime}\right)=6$. But $\tilde{M}_{2} \cap M^{\prime}$ is isomorphic to $\operatorname{Hom}\left({ }_{M} \mathbb{L}^{2}\left(\tilde{M}_{1}\right)_{M}\right)$ and hence this is not the case. So (i) holds and $G$ is not commutative i.e. $G \cong S_{3}$. From $[N: \mathbb{L}]=2$, there exists $\tau$ an outer action of $\mathbb{Z}_{2}$ on $L$ such that

$$
N=L \underset{\tau}{\rtimes} \mathbb{Z}_{2}
$$

Then $\tau$ is implemented by a unitary in $M$. [PP3, Proposition 1.7 (ii)] shows that $\tau$ comes from a subgroup action of $G$ up to cocycle conjugacy, and so we obtain the result.

Q.E.D.

\section{References}

[A] Aubert, P., Théorie de Galoi pour une $W^{*}$ algebre, Comment. Math. Helv. 39 (51), (1976), 411-433.

[C] Connes, A., Periodic automorphisms of the hyperfinite factor of type $\mathrm{II}_{1}$, Acta Sci. Math., 39, (1977), 39-66.

[G] Goldman, M., On subfactors of factors of type $\mathrm{II}_{1}$, Mich. Math. J., 7 (1960) 167-172.

[GHJ] Goodman, F., De la Harpe, P., Jones, V., Coxeter graphs and towers of algebras, MSRI Publications 14, Springer Verlag, Berlin, Heidelberg, New York, 1989.

[H] Hiai, F., Minimizing indices of conditional expectations on a subfactor, Publ. RIMS, Kyoto Univ., 24 (1988), 673-678.

[I] Izumi, M., Application of fusion rules to classification of subfactors, Publ. RIMS, Kyoto Univ., 27 (1991), 953-994.

[J1] Jones, V., Index for subfactors, Invent. Math., 72 (1983), 1-25.

[J2] —, Index for subrings, Contemp. Math., 43 (1985), 181-190.

[K1] Kosaki, H., Extension of Jones theory on index to arbitrary factors, J. Funct. Anal., 66 (1986), 123-140.

[K2] - Characterization of crossed product (properly infinite case), Pacific J. Math., 137 (1989), 159-167.

[KL] Kosaki, H., and Longo, R., A remark on the minimal index of subfactors, J. Funct. Anal., 107 (1992), 458-470.

[Kw] Kawahigashi, Y., Automorphism commuting with a conditional expectation onto a subfactor with finite index, to appear in J. Oper. Theory.

[L1] Longo, R., Index of subfactors and statistics of quantum fields I, Commun. Math. Phys., 126 (1989), 217-247.

[L2] - Index of subfactors and statistics of quantum fields II, Commun. Math. Phys., 130 (1990), 285-309.

[L3] - Simple injective subfactors, Adv. Math., 63 (1987), 152-171.

[L4] -, Minimal index and braided subfactors, J. Funct. Anal., 109 (1992), 98-112.

[Li] Loi, P., On automorphisms of subfactors, preprint.

[O1] Ocneanu, A., Quantized group string algebra and Galois theory for algebra, in Operator algebras and applications, Vol. 2 (Warwick, 1987), London Math. Soc. Lect. Note 
Series Vol. 136, Cambridge University Press, (1988), 119-172.

[O2] Ocneanu, A., Quantum symmetry, differential geometry of finite graphs and classification of subfactors, University of Tokyo Seminary Notes, (Notes recorded by Y. Kawahigashi), 1990.

[P1] Popa, S., Classification of subfactors: reduction to commuting squares, Invent. Math., 101 (1990), 19-43.

[P2] - Correspondences, preprint.

[PP1] Pimsner, M., and Popa, S., Finite dimensional approximation of algebras and obstruction for index, J. Funct. Anal., 98 (1991), 270-291.

[PP2] - - Interating the basic construction, Trans. Amer. Math. Soc., 310 (1988), 127-133.

[PP3] - Entropy and index for subfactors, Ann. sient. Éc. Norm. Sup., 4 (1986), 57-106.

[S] Sauvageot, J. L., Sur le produit tensoriel relatif de'espaces de Hilbert, J. Oper. Th., 9 (1983), 237-252. 
